\title{
Degree of endplate chondrocyte degeneration in different tension regions during mechanical stimulation
}

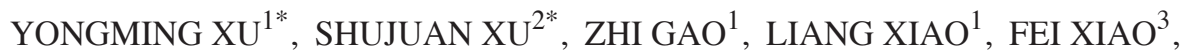 \\ HONGGUANG XU ${ }^{1}$ and XIAOLING ZHANG ${ }^{3,4}$
}

\begin{abstract}
Departments of ${ }^{1}$ Orthopedic Surgery and ${ }^{2}$ Surgery, Yijishan Hospital, The First Affiliated Hospital of Wannan Medical College, Wuhu, Anhui 241001; ${ }^{3}$ Department of Orthopedic Surgery, Xinhua Hospital Affiliated to Shanghai Jiao Tong University School of Medicine, Shanghai 200011; ${ }^{4}$ Key Laboratory of Stem Cell Biology, Institute of Health Sciences, Shanghai Jiao Tong University School of Medicine and Shanghai Institutes for Biological Sciences, Chinese Academy of Sciences, Shanghai 200031, P.R. China
\end{abstract}

Received May 15, 2017; Accepted November 16, 2017

DOI: $10.3892 / \mathrm{mmr} .2018 .8435$

\begin{abstract}
The aim of this study was to explore the degree of degeneration of endplate chondrocytes in different tension regions induced by intermittent cyclic mechanical tension (ICMT) in vitro. Rat endplate chondrocytes were harvested and treated with $10 \%$ ICMT for $8 \mathrm{~h}$ /day with a frequency of $0.5 \mathrm{~Hz}$. A cartilage degeneration model was induced using an FX-5000T cell strain-loading system. The experiment was divided into the central region and the peripheral region, according to the contact area between the loading post and the six-well flexible silicone rubber BioFlex plates. Toluidine blue and phalloidin staining were used to observe the morphological changes of cells following mechanical stimulation. Apoptosis was detected by flow cytometry and the mRNA and protein expression levels of collagen type II $\alpha 1$, aggrecan, SRY-box 9 and matrix metalloproteinase 13 were detected by reverse transcription-quantitative polymerase chain reaction (RT-qPCR) and western blotting, respectively. Endplate chondrocytes exhibited degenerative alterations under mechanical conditions of $10 \%$ ICMT and $0.5 \mathrm{~Hz}$ at $8 \mathrm{~h}$ /day. Toluidine blue and phalloidin staining demonstrated that the cells in the
\end{abstract}

Correspondence to: Professor Hongguang $\mathrm{Xu}$, Department of Orthopedic Surgery, Yijishan Hospital, The First Affiliated Hospital of Wannan Medical College, 2 Zheshan West Road, Wuhu, Anhui 241001, P.R. China

E-mail:xuhg@medmail.com.cn

Professor Xiaoling Zhang, Department of Orthopedic Surgery, Xinhua Hospital Affiliated to Shanghai Jiao Tong University School of Medicine, 1665 Kongjiang Road, Shanghai 200011, P.R. China

E-mail: xlzhang@sibs.ac.cn

${ }^{*}$ Contributed equally

Key words: FX-5000T tension system, endplate chondrocytes, intermittent cyclic mechanical tension, chondrocyte degeneration, cell morphology peripheral region were more slender compared with cells in the central region, but RT-qPCR and western blotting results demonstrated that the degree of cell degeneration between the two groups was not statistically differences. So that cell morphological alteration does not imply that cells have undergone degeneration.

\section{Introduction}

Intervertebral disc degeneration (IVDD) has been associated with a number of factors, including mechanical loading, aging, genetic factors and nutrition. IVDD is considered to be an important factor for neck and back pain (1). The age of occurrence of IVDD is becoming younger as current changes in human lifestyles ultimately affect daily life and work (2). Although medication or surgical treatment may be able to eliminate some of the symptoms, it is still difficult to radically prevent or stop the progression of IVDD (2). IVDD manifests extensive histomorphological alterations, including fibrosis of the nucleus pulposus, disorganization of the annulus fibrosus lamellae and thinning and calcification of the cartilage end plate (1). However, the underlying mechanism of pathophysiology of the disc degenerative process remains to be elucidated (3). Therefore, it is important to determine the pathophysiological changes of IVDD progression. Intervertebral discs acquire nutrition mainly through the endplate pathway, and the protection of endplate cartilage may effectively delay the occurrence of IVDD (2). An intervertebral disc is located between two neighboring vertebral bodies and functions to buffer the tension induced by body weight, tensile force and shear force generated by spinal activity (3). Therefore, external mechanical stimulation is regarded as one of the most important factors leading to IVDD (4). In our previous study, the synthesis, deposition and degradation of the extracellular matrix (ECM) components of endplate chondrocytes were demonstrated to change following applied intermittent cyclic mechanical tension in vitro, which subsequently led to the degeneration of endplate chondrocytes (5). SRY box 9 (SOX9) is a transcription factor regulating the synthesis of extracellular matrix (5). Collagen type II $\alpha 1$ (COL2A1) and aggrecan 
(ACAN) are components in the extracellular matrix, and matrix metalloproteinase 13 (MMP13) is a marker of cartilage degeneration (5). SOX9, COL2A1 and ACAN alleviates IVDD, while MMP13 promotes the progression of IVDD (5).

The present study used an FX-5000T tension system to effectively simulate the stress condition of cells in vitro. This system has been widely used in research on various tissues, including bone healing, cartilage cells, periodontal ligament cells, lung epithelial cells, myocardial cells, skin cells and tendon cells (6-10). The present study aimed to clarify the relationship between chondrocyte morphological changes and the degree of degeneration, which may provide insight for the examination of related diseases that are induced by mechanical stimulation.

\section{Materials and methods}

Primary culture of rat endplate chondrocytes. A total of 12 (2-month-old, male, weight, 150-180 g) Sprague-Dawley rats were purchased from Shanghai SLAC Laboratory Animal Co., Ltd. (Shanghai, China) and were individually caged under standard laboratory conditions $\left(12 \mathrm{~h} \mathrm{light} /\right.$ dark cycle, $21^{\circ} \mathrm{C}$ controlled temperature, relative humidity $40-70 \%$, free access to food and water, $0.03 \% \mathrm{CO}_{2}$ ). All experimental procedures were approved by the Committees of the Yijishan Hospital, The First Affiliated Hospital of Wannan Medical College. Endplate cartilage was obtained from Sprague-Dawley rats that were anesthetized by intraperitoneal injection with $4 \%$ chloral hydrate $(500 \mathrm{mg} / \mathrm{kg})$ and sacrificed by cervical dislocation. The whole lumbar spine was removed and washed twice with $1 \mathrm{X}$ PBS, containing $1 \times 10^{8} \mathrm{U} / 1$ penicillin and $1 \mathrm{~g} / \mathrm{l}$ streptomycin. The annulus fibrous and nucleus pulposus were incised to expose the shallow disc-like translucent endplate cartilage, which was subsequently cut into a small piece $\left(1 \mathrm{~mm}^{3}\right)$ with ophthalmic scissors. Chondrocytes were obtained by digestion with trypsin and collagenase II (Gibco; Thermo Fisher Scientific, Inc., Waltham, MA, USA), as previously described (11). The cells were washed twice with $1 \mathrm{X}$ PBS and cultured in a $10 \mathrm{~cm}$ Petri dish with Dulbecco's modified Eagle's medium (DMEM)/F-12 medium (HyClone; GE Healthcare Life Sciences, Logan, UT, USA) supplemented with $10 \%$ fetal bovine serum (FBS: Gibco; Thermo Fisher Scientific, Inc.) in a humidified incubator containing $5 \% \mathrm{CO}_{2}$ at $37^{\circ} \mathrm{C}$. Culture medium was replaced every three days and endplate chondrocytes were collected at the third passage (P3). This study was approved by the Research Ethics Committee of Yijishan Hospital affiliated to Wannan Medical College (Wuhu, China).

Application of intermittent cyclic mechanical stretch. P3 rat endplate chondrocytes were seeded $\left(2 \times 0^{5}\right.$ cells/well) in $2 \mathrm{ml} \mathrm{DMEM} / \mathrm{F}-12$ medium with $10 \% \mathrm{FBS}$ on 6 -well flexible silicone rubber BioFlex plates that were covered with collagen type I (Flexcell International Corporation, Burlington, NC, USA), and incubated at $37^{\circ} \mathrm{C}$ in a humidified incubator containing $5 \% \mathrm{CO}_{2}$. At $70-80 \%$ confluence, cells in the intermittent cyclic mechanical tension (ICMT) group received mechanical loading $(0.5 \mathrm{~Hz}$ sinusoidal curve; $10 \%$ elongation; $8 \mathrm{~h}$ /day) that was applied using the FX-5000T cell stretch tensile stress loading system (Flexcell International
Corporation); cells in the negative control (NC) group did not receive mechanical loading. The FX-5000T cell stretch tensile stress loading system was manufactured by Flexcell International Corporation (Fig. 1). The basic principle of the system (negative pressure exchange mode) is to form an effective closed cavity in the cell culture plate between the basement membrane and the substrate through a rubber gasket. The inlet and outlet of the sealed chamber are inserted into the $\mathrm{CO}_{2}$ incubator and the inlet and outlet pipes are connected to the sealed chamber in the incubator. The vacuum pump is used to extract the negative pressure generated by the enclosed chamber to pull the three-dimensional top column to cause the deformation of the elastic base film. The deformation of the basement membrane is altered by the computer control system to monitor the pressure of the regulating gas and the adherent growing cells are subjected to stretch loading mechanic stimulus. Owing to the loading system gas pressure adjustment of the top pillar and the BioFlex six-hole multi-directional plate not being in full contact as presented in Fig. 1C, with the extension of mechanical stimulation time, the morphologies of cells in the central and periphery regions were different.

Flow cytometry. P3 endplate chondrocytes were seeded ( $2 \times 10^{5}$ cells/well) on 6-well flexible silicone rubber BioFlex plates coated with collagen I. ICMT $(0.5 \mathrm{~Hz}$ sinusoidal curve; $10 \%$ elongation; $8 \mathrm{~h}$ /day) was applied when the cells reached $70-80 \%$ confluence for 1 day and 2 days. The membrane of the six-well flexible silicone rubber BioFlex plates was cut along the dotted line (Fig. 1) following the applied mechanical loading. Cells in the central and peripheral region of each well were separately scraped with a cell scraper and collected by centrifugation following two washes with cold PBS. The supernatant was removed and $1 \mathrm{X}$ Annexin-binding buffer (Thermo Fisher Scientific, Inc.) was added to resuspend the cells at a density of $1 \times 10^{6}$ cells/ml. Annexin V-Alexa Fluor $488(5 \mu \mathrm{l}$; Thermo Fisher Scientific, Inc.) and propidium iodide (PI; $1 \mu \mathrm{l}$; $100 \mu \mathrm{g} / \mathrm{ml}$ ) working solution (Thermo Fisher Scientific, Inc.) were added to each $100 \mu \mathrm{l}$ of the cells suspension, and $200 \mu \mathrm{l}$ of $1 \mathrm{X}$ Annexin-binding buffer was added following incubation at room temperature for $15 \mathrm{~min}$. Unless indicated, all of the above procedures were maintained on ice. Stained cells were immediately analyzed by flow cytometry using a FACSCalibur flow cytometer (BD Biosciences, San Jose, CA, USA) with FACSDiva software (BD Biosciences; v 8.0.1). PI and annexin V-Alexa Fluor 388 double-positive cells were defined as apoptotic.

Toluidine blue staining. ICMT cells received mechanical loading for 3 days. The cells were collected and washed twice with PBS, fixed with $4 \%$ paraformaldehyde for $15 \mathrm{~min}$ at $25^{\circ} \mathrm{C}$ followed by 2 washes with PBS. Subsequently, the cells were stained with $0.5 \%(\mathrm{~W} / \mathrm{W})$ toluidine blue for $30 \mathrm{~min}$ at $25^{\circ} \mathrm{C}$. An light inverted microscope was used to capture images (5 fields/slide) following removal of the staining dye.

Phalloidin cytoskeleton staining. P3 endplate chondrocytes were seeded ( $1 \times 10^{3}$ cells/well; 6-well plates) on confocal glass culture dishes and grown until $40-50 \%$ confluent at $37^{\circ} \mathrm{C}$. The culture medium was removed and the cells were washed twice 
with PBS and fixed in $4 \%$ paraformaldehyde solution at room temperature for $15 \mathrm{~min}$. Cells were washed twice with PBS and permeabilized in 5\% Triton X-100 solution (Yeasen, Shanghai, China) for $10 \mathrm{~min}$ at $25^{\circ} \mathrm{C}$. Following three washes with PBS, fluorescein isothiocyanate (FITC)-labeled phalloidin solution $(200 \mu \mathrm{l}$; Yeasen) was added to each well and cells were incubated in the dark at room temperature for $30 \mathrm{~min}$. Nuclei were stained with DAPI solution (200 $\mu \mathrm{l}$; Yeasen) for $30 \mathrm{sec}$ at $25^{\circ} \mathrm{C}$. Images were captured using a laser confocal microscope with FITC excitation (Ex) and emission (Em) filters at 496 and $516 \mathrm{~nm}$, respectively, and DAPI Ex and Em at 364 and $454 \mathrm{~nm}$, respectively.

RNA extraction and reverse transcription-quantitative polymerase chain reaction $(R T-q P C R)$. Cells in the central and peripheral regions of the BioFlex plates were collected separately, as aforementioned. Total RNA was isolated using TRIzol reagent (Invitrogen; Thermo Fisher Scientific, Inc.), according to the manufacturer's instructions. First-strand cDNA was synthesized from $1 \mu \mathrm{g}$ of total RNA by incubating for $1 \mathrm{~h}$ at $42^{\circ} \mathrm{C}$ with Superscript III reverse transcriptase (Invitrogen; Thermo Fisher Scientific, Inc.) following oligo (dT) priming. Following reverse transcription, qPCR was performed using a LightCycler 480 Real-Time PCR System (Roche Diagnostics, Basel, Switzerland) using SYBR1 Premix Ex Taq (Takara Biotechnology Co., Ltd., Dalian, China), according to the manufacturer's protocol. Primer sequences are provided in Table I; GAPDH was used as internal control. qPCR thermocycling conditions were as follows: Initial denaturation at $95^{\circ} \mathrm{C}$ for $10 \mathrm{sec}, 50$ cycles at $95^{\circ} \mathrm{C}$ for $10 \mathrm{sec}$ and $60^{\circ} \mathrm{C}$ for $30 \mathrm{sec}$; and final extension at $95^{\circ} \mathrm{C}$ for $15 \mathrm{sec}$ and $60^{\circ} \mathrm{C}$ for $30 \mathrm{sec}$. The data were analyzed using the comparison $2^{-\Delta \Delta \mathrm{Cq}}$ method and expressed as the fold change relative to the respective control (12). Each sample was analyzed in triplicate.

Western blotting. Endplate chondrocytes received mechanical stimulation for 7 days, and were collected as aforementioned. Protein was extracted from the cells $\left(1 \times 10^{4}\right.$ cells/well; 6-well plates) following cell lysis with $100 \mu \mathrm{l}$ radioimmunoprecipitation buffer containing $1 \%$ phenylmethanesulfonyl, then the cell lysates were placed on ice for $30 \mathrm{~min}$ followed by a high-speed centrifuge at $13,500 \mathrm{x}$ g for $15 \mathrm{~min}$ at $4^{\circ} \mathrm{C}$. The supernatant was collected, and protein concentration was determined using a BCA Protein Assay kit. The protein was denatured with loading buffer and incubated at $95^{\circ} \mathrm{C}$ for $15 \mathrm{~min}$. A total of $20 \mu \mathrm{g}$ protein was loaded per lane using 10\% SDS-PAGE and electrotransferred onto nitrocellulose membranes (Whatman; GE Healthcare Life Sciences). Membranes were blocked with $5 \%$ bovine serum albumin (BIOSHARP Technology, Inc., Shanghai, China) for $1 \mathrm{~h}$ at $25^{\circ} \mathrm{C}$ and incubated overnight at $4^{\circ} \mathrm{C}$ with the following primary antibodies (all at 1:800): Monoclonal rabbit anti-GAPDH (catalog no. 14C10; Cell Signaling Technology, Inc., Danvers, MA, USA), polyclonal rabbit anti-SRY box 9 (SOX9; catalog no. CY5400; Shanghai Abways Biotechnology Co., Ltd., Shanghai, China), polyclonal rabbit anti-matrix metalloproteinase 13 (MMP13; catalog no. AY3010; Shanghai Abways Biotechnology Co., Ltd.) and monoclonal rabbit anti-collagen type II $\alpha 1$ (COL2A1; catalog
Table I. Primer sequences for reverse transcription-quantitative polymerase chain reaction.

\begin{tabular}{llc}
\hline Gene & \multicolumn{1}{c}{ Primer sequence $\left(5^{\prime} \rightarrow 3^{\prime}\right)$} & $\begin{array}{c}\text { Length } \\
(\mathrm{bp})\end{array}$ \\
\hline SOX9 & $\begin{array}{l}\text { F: CGTCAACGGCTCCAGCA } \\
\text { R: TGCGCCCACACCATGA }\end{array}$ & 69 \\
COL2A1 & F: CCTGAAACTCTGCCACCCAG & 151 \\
& R: GTTCTTCCGAGGCACAGTCG & \\
ACAN & F: ACACCCCTACCCTTGCTTCT & 124 \\
& R: AAAGTGTCCAAGGCATCCAC & \\
MMP13 & F: CCCTGGAGCCCTGATGTTT & 109 \\
& R: CTCTGGTGTTTGGGGTGCT & \\
GAPDH & F: CTCAACTACATGGTCTACATGT & 81 \\
& TCCA & \\
& R: TGCGCCCACACCATGA & \\
\hline
\end{tabular}

ACAN, aggrecan; COL2A1, collagen type II $\alpha 1$; F, forward; MMP13, matrix metalloproteinase 13; R, reverse; SOX9, SRY-box 9.

no. P02458; Bioworld Technology, Inc., St. Louis Park, MN, USA). The membranes were washed three times with TBS with $0.5 \%$ Tween-20 (TBST) and incubated with horseradish peroxidase-conjugated goat anti-rabbit immunoglobulin G secondary antibodies (catalog no. 7074; Cell Signaling Technology, Inc., Danvers, MA, USA) were used at a 1:5,000 dilution at room temperature for $2 \mathrm{~h}$. The antigen-antibody complexes were visualized with the enhanced chemiluminescence detection system (EMD Millipore, Billerica, MA, USA). Immunoreactive bands were quantified on autoradiography films in triplicate with using Image $\mathrm{J}$ software (version 4.8; National Institutes of Health, Bethesda, MD, USA) by normalizing the band intensities to GAPDH.

Statistical analysis. All data are expressed as the mean \pm standard error of the mean and analyzed by one-way analysis of variance followed by Bonferroni post hoc test as appropriate. All tests were two-tailed; $\mathrm{P}<0.05$ was considered to indicate a statistically significant difference. All statistical analyses were conducted with SPSS 18.0 (SPSS Inc., Chicago, IL, USA).

\section{Results}

Degeneration of rat endplate chondrocytes induced in vitro. RT-qPCR results revealed that the mRNA expression levels of cartilage-related anabolic genes SOX9, COL2Al and ACAN in the ICMT-treatment group gradually decreased on days 1 , 3 and 7 compared with NC (Fig. 2A-C), whereas the expression levels of MMP13 gradually increased compared with NC at the same time points (Fig. 2D). Rat endplate chondrocytes exhibited degenerative alterations following ICMT including decreased expression of SOX9, COL2A1 and ACAN and elevated expression of MMP13.

Cell morphological changes in the central and peripheral regions. Rat chondrocytes were treated with mechanical 

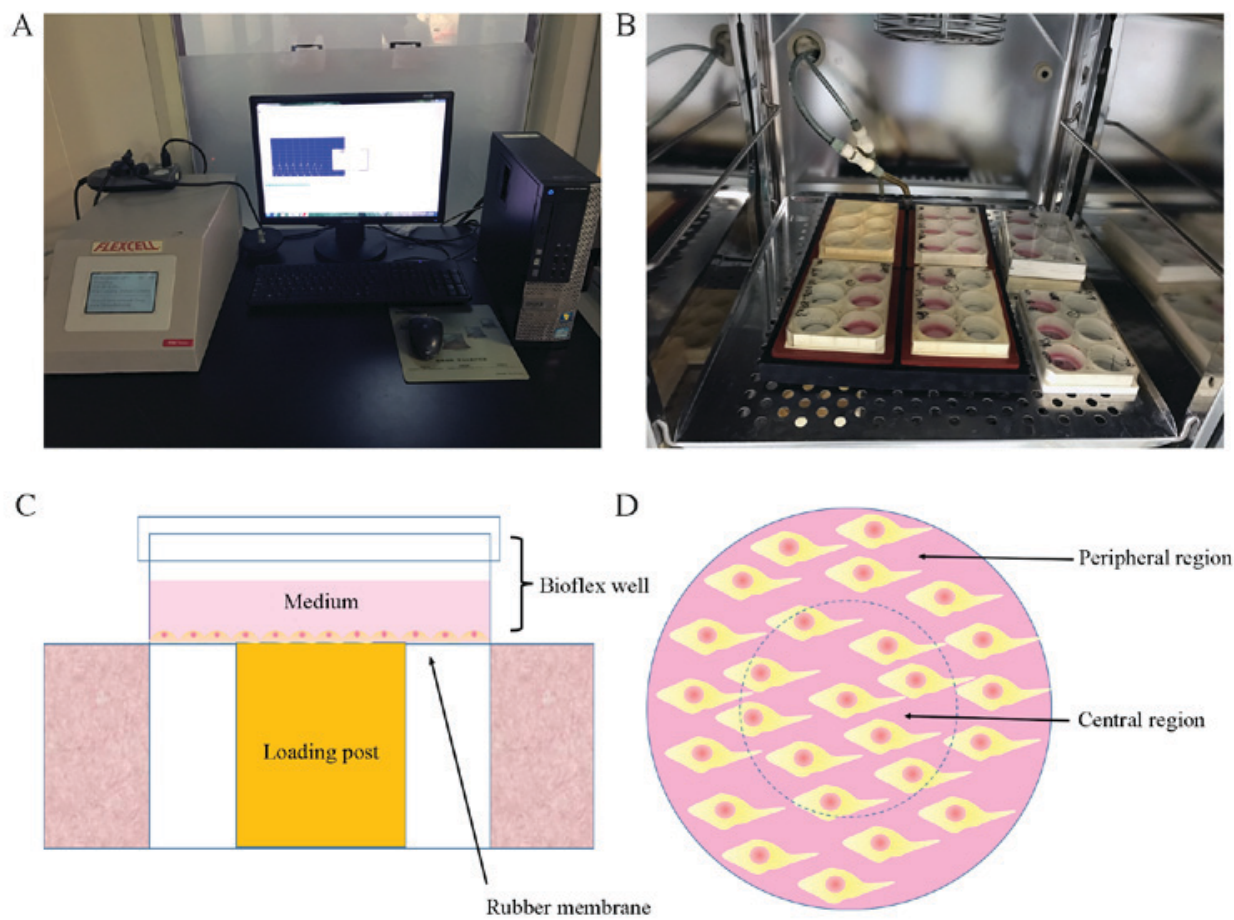

D

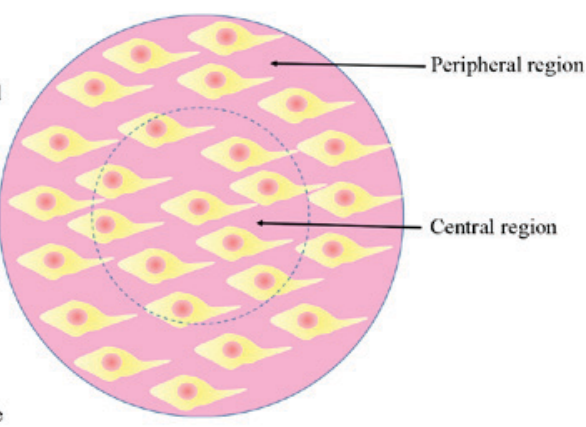

Figure 1. FX-5000T system. (A) Computer monitoring system. (B) Six-well plates. (C) Schematic of six-well plates under mechanical loading. (D) Top view of six-well plates, the inner area of the dotted line represents the central region and the outer area is the peripheral region.

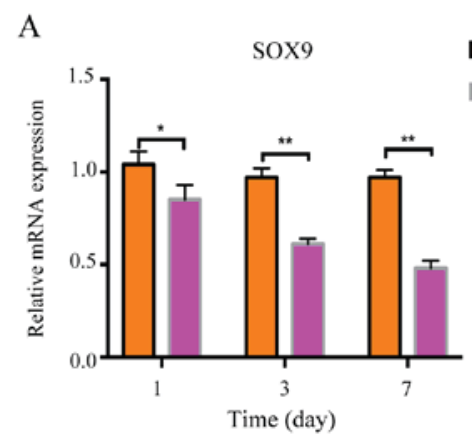

$\mathrm{C}$

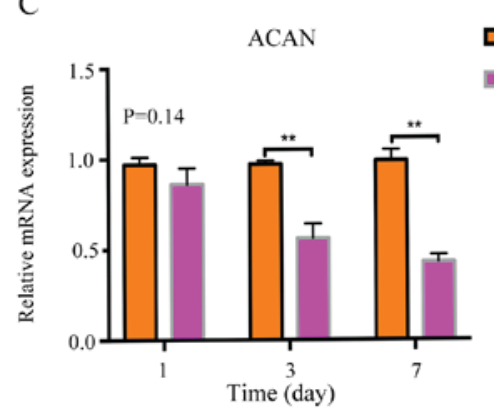

B
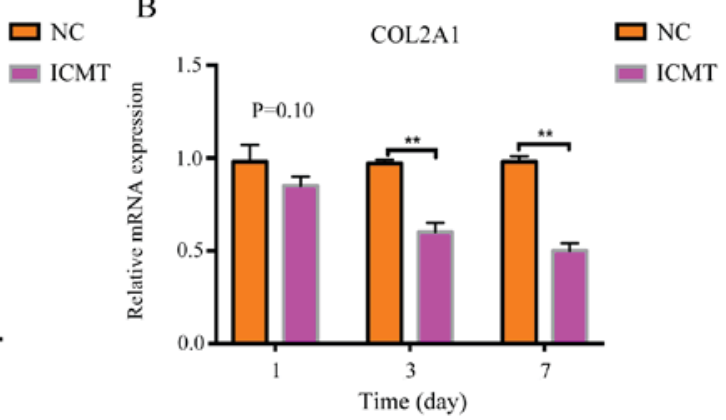

$\mathrm{D}$
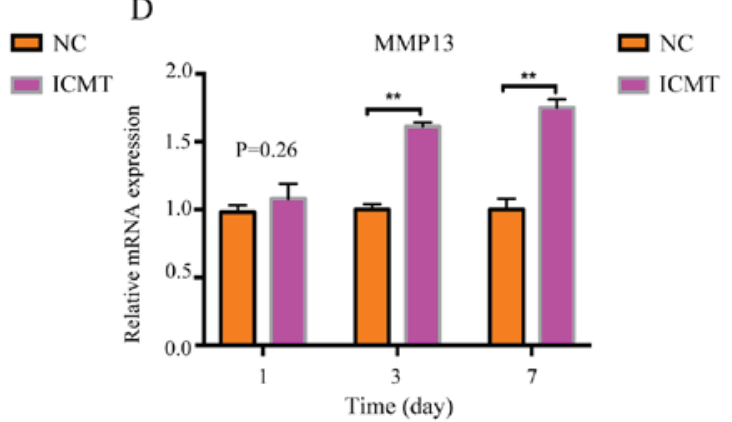

Figure 2. ICMT stimulation induces endplate cartilage degeneration. Relative mRNA expression levels of chondrocyte-related genes (A) SOX9, (B) COL2A1, (C) ACAN and (D) MMP13 were measured following ICMT stimulation and compared with unstimulated NC. "P<0.05 and ${ }^{* * *} \mathrm{P}<0.01$ vs. NC. ACAN, aggrecan; COL2A1, collagen type II $\alpha 1$; ICMT, intermittent cyclic mechanical tension; MMP13, matrix metalloproteinase 13; NC, negative control; SOX9, SRY-box 9.

stimulation (10\% ICMT, $0.5 \mathrm{~Hz}$ and $8 \mathrm{~h} /$ day) for 3 days. Toluidine blue staining demonstrated a long shuttle change of cells in the peripheral region of the rubber membrane compared with cells in the central region (Fig. 3A-C). Phalloidin cytoskeleton staining also revealed that the filamentous (F)-actin in the peripheral cells were more notably stretched and elongated compared with the F-actin in cells in the central region as indicated by the red arrow (Fig. 3D and E).

Effects of ICMT on apoptosis in the central and peripheral regions. The levels of apoptosis were also examined to further determine the effects of ICMT induction on endplate chondrocytes; cells received mechanical stimulation for 

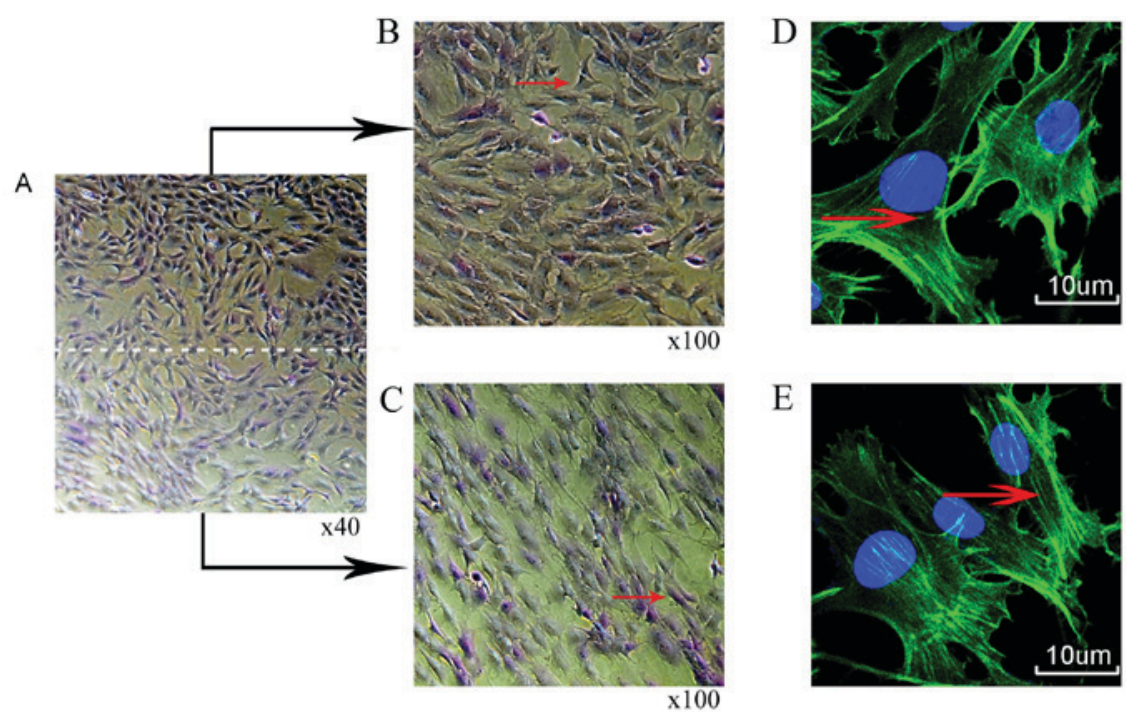

Figure 3. Morphological changes of chondrocytes following mechanical stimulation for 3 days. (A) Toluidine blue staining (B) central and (C) peripheral regions. Phalloidin cytoskeleton staining (D) central and (E) peripheral regions; green indicates filamentous-actin; blue indicates DAPI stained nuclei.

$24 \mathrm{~h}$
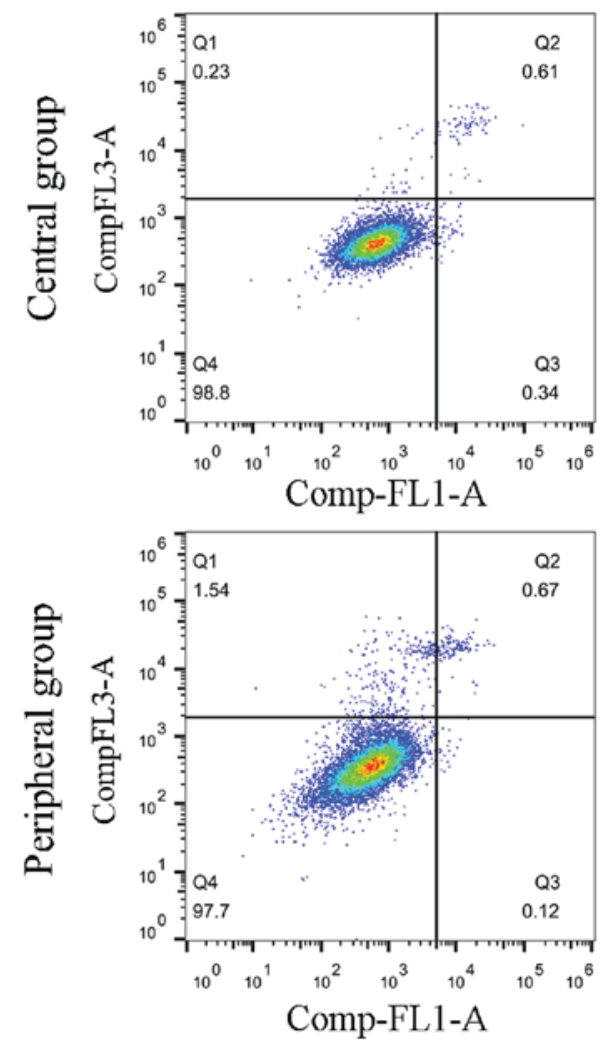

$48 \mathrm{~h}$
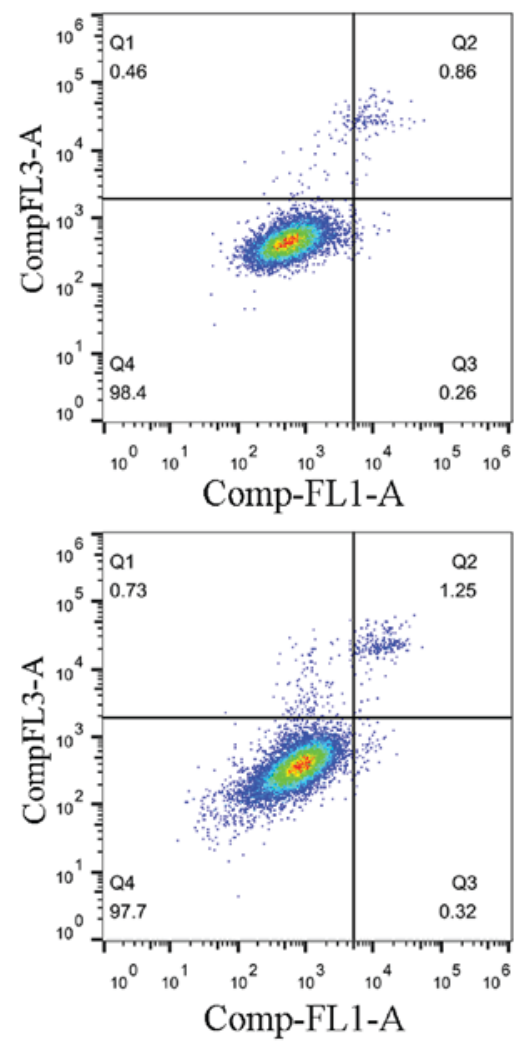

Figure 4. Endplate chondrocytes were subjected to ICMT for 24 and 48 h, and cell apoptosis was examined by flow cytometry. No notable differences were observed between cells in the central and peripheral regions at 24 or 48 h. ICMT, intermittent cyclic mechanical tension; Comp-FL1-A: annexin V-Alexa Fluor 488 and Comp-FL3-A: PI.

24 and $48 \mathrm{~h}$. No differences were identified for the levels of apoptosis between the ICMT and NC groups at either 24 or 48 h (Fig. 4).

Degree of cell degeneration between the central and peripheral regions. Results from RT-qPCR indicated that there were no significant differences in the mRNA expression levels of the cartilage-related genes, SOX9, COL2A1, ACAN and MMP13, between cells in the central and peripheral regions following mechanical stimulation (Fig. 5). Similarly, western blotting results indicated no notable differences in SOX9, COL2A1 and MMP13 protein expression levels between cells in the two regions, however, due to the lack of a suitable ACAN antibody, alterations in protein expression of ACAN were not detected 

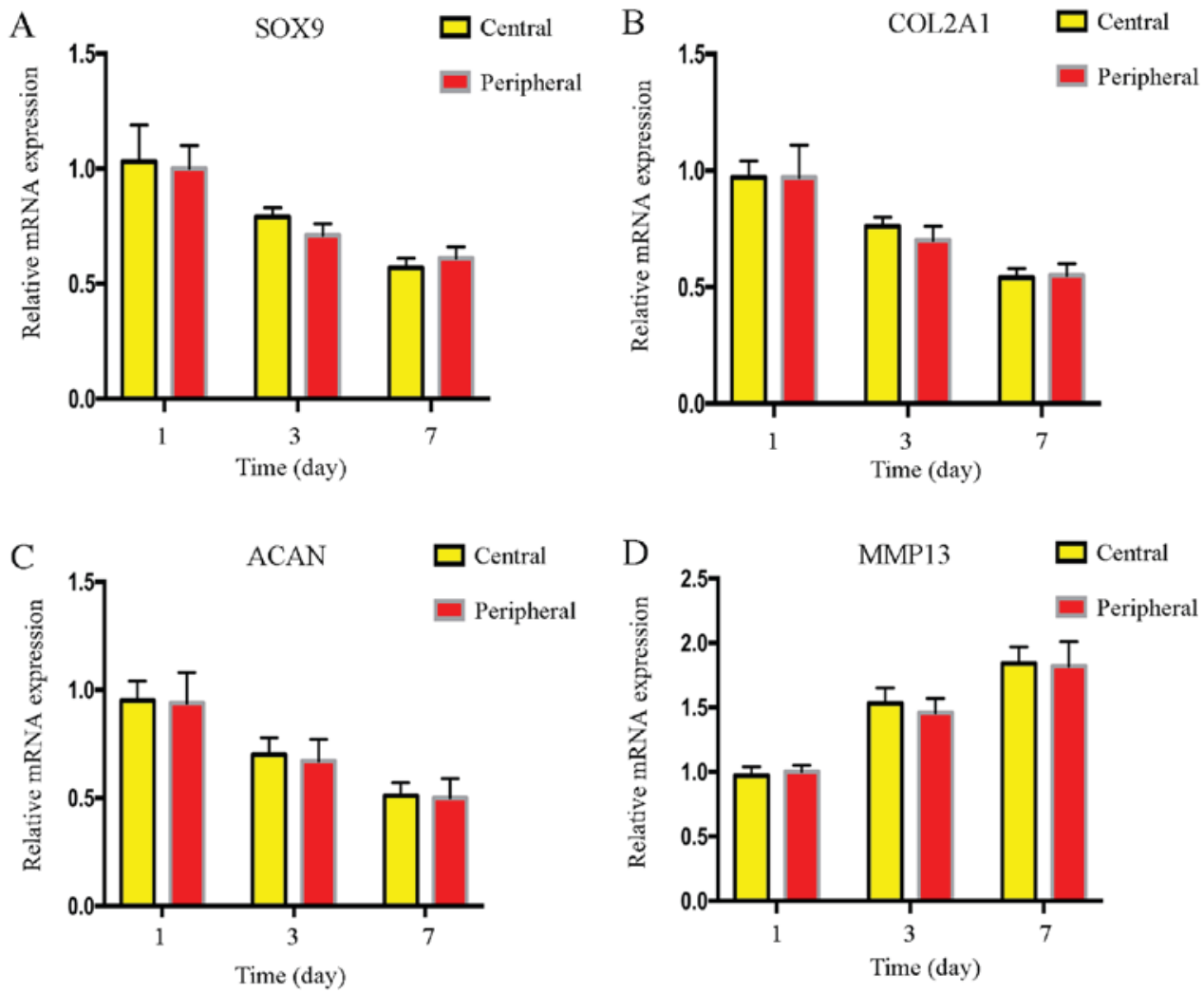

Figure 5. mRNA expression level changes of endplate chondrocytes between cells in the central and peripheral groups following mechanical stimulation. The mRNA expression levels of chondrocyte-related genes (A) SOX9, (B) COL2A1, (C) ACAN and (D) MMP13 were not significantly different between the two groups at days 1, 3 and 7 . ACAN, aggrecan; COL2A1, collagen type II $\alpha 1$; ICMT, intermittent cyclic mechanical tension; MMP13, matrix metalloproteinase 13; NC, negative control; SOX9, SRY-box 9.

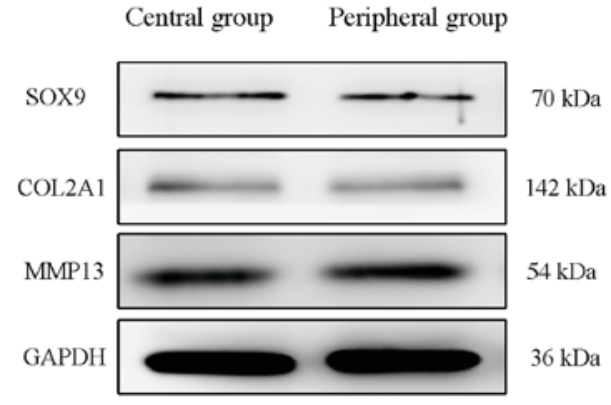

Figure 6. Expression levels of endplate chondrocyte-associated proteins in central and peripheral cells following mechanical stimulation. Western blot analysis revealed no notable differences in the protein expression levels of SOX9, COL2A1 and MMP13 between the two groups. COL2A1, collagen type II a1; MMP13, matrix metalloproteinase 13; SOX9, SRY-box 9.

(Fig. 6). There was no difference in the degree of the endplate chondrocyte degeneration between the two groups.

\section{Discussion}

Endplate cartilage is a thin layer of hyaline cartilage that lies between the vertebrae and the intervertebral disc, and mainly comprises chondrocytes and ECM. Endplate cartilage effectively regulates the mechanical stimulation in the course of physiological activities (13). Excessive mechanical stimulation may cause metabolic imbalances of the cartilage ECM through the relevant signaling pathways, including Bone morphogenetic proteins (BMPs) and transforming growth factor- $\beta$ (TGF- $\beta$ ), and may result in lower back pain and neurological dysfunction $(14,15)$. The ECM of endplate cartilage is mainly composed of collagen fibers and cartilage proteoglycans; the main collagen fiber is type II collagen (16). It was previously reported that the ECM components of endplate chondrocytes were altered following mechanical stimulation, and the cytoskeleton became more slender $(7,17)$. The cytoskeleton consists of microfilaments, microtubules and intermediate filaments, comprising actin, tubulin and vimentin. In chondrocytes, actin filaments function to maintain the chondrocyte phenotype by modulating cell shape and to provide mechanical integrity to withstand compression $(18,19)$. A previous study demonstrated that mechanical stimulation may lead to alterations in the structure and morphology of intracellular actin (20); it was confirmed that cell degeneration is closely related to cytoskeletal actin, but the specific mechanism remains unknown.

Results from the presents study demonstrated a change in cell morphology in different regions (central and peripheral) following mechanical stimulation. Immunocytochemical staining demonstrated that the cells in the peripheral region were particularly elongated following ICMT, and that the F-actin cytoskeleton was stretched and elongated, which indicated that mechanical stimulation caused notable morphological changes of the cytoskeleton protein actin. RT-qPCR and western blotting results demonstrated that the mRNA and protein expression levels, respectively, of the 
cartilage-related genes SOX9, COL2A1, ACAN and MMP13 were not different between cells in the central and peripheral regions following mechanical stimulation, which indicated that the degree of cell degeneration induced by mechanical stimulation between the two groups was consistent. In addition, although the morphological changes between the two regions were different, no differences in apoptosis between the two groups were observed following stimulation for 24 and $48 \mathrm{~h}$.

A previous study demonstrated that actin in the cytoskeleton regulates the metabolism of the ECM through relevant mechanical signaling pathway, including BMPs and TGF- $\beta$, and ultimately induces changes in chondrocyte degeneration (21). In the present study, the morphological changes of cells did not necessarily coincide with the degree of cell degeneration. Although these results differed from previous studies, it was demonstrated that cell morphological changes may occur 3 days following mechanical stimulation. The present study was not able to validate the relationship between cell morphological changes and degeneration by extending the loading time, which requires further investigation. It is proposed to induce the degeneration of chondrocytes in a short time by changing the intensity of mechanical stimulation. Further study on the intrinsic relationship between cell morphology and cell degeneration may improve our understanding of the pathogenesis of intervertebral disc degeneration disease and may provide a more comprehensive theoretical and experimental basis for the study of intervertebral disc degeneration caused by mechanical factors.

\section{Acknowledgements}

The present study was supported by a grant from The National Natural Science Foundation of China (grant no. 81572185), The Natural Science Foundation of Anhui Province of China (grant no. 1708085MH185) and The Scientific and Technological Achievements Transformation Project of Wuhu City (grant no. 2017CG27).

\section{References}

1. Manek NJ and MacGregor AJ: Epidemiology of back disorders: Prevalence, risk factors, and prognosis. Curr Opin Rheumatol 17: 134-140, 2005.

2. Rodriguez AG, Rodriguez-Soto AE, Burghardt AJ, Berven S, Majumdar S and Lotz JC: Morphology of the human vertebral endplate. J Orthop Res 30: 280-287, 2012.

3. Walter BA, Korecki CL, Purmessur D, Roughley PJ, Michalek AJ and Iatridis JC: Complex loading affects intervertebral disc mechanics and biology. Osteoarthritis Cartilage 19: 1011-1018, 2011.

4. Vergroesen PP, Kingma I, Emanuel KS, Hoogendoorn RJ, Welting TJ, van Royen BJ, van Dieën JH and Smit TH: Mechanics and biology in intervertebral disc degeneration: A vicious circle Osteoarthritis Cartilage 23: 1057-1070, 2015.
5. Xu HG, Yu YF, Zheng Q, Zhang W, Wang CD, Zhao XY, Tong WX, Wang H, Liu P and Zhang XL: Autophagy protects end plate chondrocytes from intermittent cyclic mechanical tension induced calcification. Bone 66: 232-239, 2014.

6. Pan J, Wang T, Wang L, Chen W and Song M: Cyclic strain-induced cytoskeletal rearrangement of human periodontal ligament cells via the Rho signaling pathway. PLoS One 9: e91580, 2014

7. Xu HG, Ma MM, Zheng Q, Shen X, Wang H, Zhang SF, Xu JJ, Wang CD and Zhang XL: P120-catenin protects endplate chondrocytes from intermittent cyclic mechanical tension induced degeneration by inhibiting the expression of RhoA/ROCK-1 signaling pathway. Spine (Phila Pa 1976) 41: 1261-1271, 2016.

8. Wang J, Wang CD, Zhang N, Tong WX, Zhang YF, Shan SZ, Zhang XL and Li QF: Mechanical stimulation orchestrates the osteogenic differentiation of human bone marrow stromal cells by regulating HDAC1. Cell Death Dis 7: e2221, 2016.

9. Zhang R, Mao P, Fu W, Pang XQ, Wang YY, Yang C, He WQ, Liu XQ and Li YM: Effects of cyclic stretch on the induction of the transdifferentiation in human lung epithelial cells. Zhonghua Wei Zhong Bing Ji Jiu Yi Xue 25: 455-459, 2013 (In Chinese).

10. Witt F, Duda GN, Bergmann C and Petersen A: Cyclic mechanical loading enables solute transport and oxygen supply in bone healing: An in vitro investigation. Tissue Eng Part A 20: 486-493, 2014.

11. Xu HG, Hu CJ, Wang H, Liu P, Yang XM, Zhang Y and Wang LT: Effects of mechanical strain on ANK, ENPP1 and TGF- $\beta 1$ expression in rat endplate chondrocytes in vitro. Mol Med Rep 4: 831-835, 2011.

12. Livak KJ and Schmittgen TD: Analysis of relative gene expression data using real-time quantitative PCR and the 2(-Delta Delta C(T)) method. Methods 25: 402-408, 2001.

13. Ferguson SJ and Steffen T: Biomechanics of the aging spine. Eur Spine J 12 (Suppl 2): S97-S103, 2003.

14. Alkhatib B, Rosenzweig DH, Krock E, Roughley PJ, Beckman L, Steffen T, Weber MH, Ouellet JA and Haglund L: Acute mechanical injury of the human intervertebral disc: Link to degeneration and pain. Eur Cells Mater 28: 98-111, 2014.

15. Sowa GA, Coelho JP, Vo NV, Pacek C, Westrick E and Kang JD: Cells from degenerative intervertebral discs demonstrate unfavorable responses to mechanical and inflammatory stimuli: A pilot study. Am J Phys Med Rehabil 91: 846-855, 2012.

16. Bruckner $P$ and van der Rest M: Structure and function of cartilage collagens. Microsc Res Tech 28: 378-384, 1994.

17. Xu HG, Zheng Q, Song JX, Li J, Wang H, Liu P, Wang J, Wang CD and Zhang XL: Intermittent cyclic mechanical tension promotes endplate cartilage degeneration via canonical Wnt signaling pathway and E-cadherin $/ \beta$-catenin complex cross-talk. Osteoarthritis Cartilage 24: 158-168, 2016.

18. Benya PD, Brown PD and Padilla SR: Microfilament modification by dihydrocytochalasin $\mathrm{B}$ causes retinoic acid-modulated chondrocytes to reexpress the differentiated collagen phenotype without a change in shape. J Cell Biol 106: 161-170, 1988.

19. Guilak F: Compression-induced changes in the shape and volume of the chondrocyte nucleus. J Biomech 28: 1529-1541, 1995.

20. Li S, Jia X, Duance VC and Blain EJ: The effects of cyclic tensile strain on the organisation and expression of cytoskeletal elements in bovine intervertebral disc cells: An in vitro study. Eur Cell Mater 21: 508-522, 2011.

21. Nofal GA and Knudson CB: Latrunculin and cytochalasin decrease chondrocyte matrix retention. J Histochem Cytochem 50: 1313-1324, 2002.

This work is licensed under a Creative Commons Attribution-NonCommercial-NoDerivatives 4.0 International (CC BY-NC-ND 4.0) License. 